\title{
Concise Total Synthesis of Biologically Interesting Prenylated Chalcone Natural Products: 4'-O-Methylxanthohumol, Xanthohumol E, and Sericone
}

\author{
Yong Rok Lee, ${ }^{*}$ Xin Li, Seung Woo Lee, Chul Soon Yong, ${ }^{\dagger}$ Ma-Ro Hwang, ${ }^{\dagger}$ and Won Seok Lyoo ${ }^{\ddagger}$ \\ School of Chemical Engineering and Technolog, Yeungnam Lniversity, Gyeongsan 71 2-749, Korea. E-mail wrlee ânu.ackr \\ College of Phamacy, Yetngnam Lniversity, Gveongsan 712-749, Korea \\ -School of Textiles, Yetngnam Liniversitw, Gyeongsan 712-749, Korea \\ Received April 2, 2008
}

\begin{abstract}
A new and efficient synthetic approach is reported for biologically interesting prenylated chalcones. f'$^{\prime} \mathrm{O}-$ methylixanthohumol (3). xanthohumol E (4). and sericone (5) from 2.4.6-trihydroxyacetophenone. The strategies involve the introduction of a prenyl group onto an aryl ring. benzopyran fonnation. and basecatalyzed aldol reactions.
\end{abstract}

Key Words : Prenylated chalcone, Xanthohumol, 4'-O-Methylxanthohumol. Xanthohumol E. Sericone

\section{Introduction}

Prenylated chalcones are an abundant subclass of flavonoids that are widely distributed in nature. ${ }^{1}$ Members of prenylated chalcones are associated with a wide variety of biological activities such as antimtalarial. ${ }^{2}$ antidiabetic. ${ }^{3}$ antifungal. ${ }^{4}$ antibacterial. ${ }^{5}$ antitumor. ${ }^{6}$ antimetastatic. ${ }^{7}$ antioxidative, anti-inflanmatory. and NF- $\kappa$ B inhibitory activities. " This wide range of biological activity has stimulated interest in the synthesis of naturally occurring prenylated chalcones. Among these, xanthohumol (1). desmethylxanthohumol (2). 4'-O-methylxanthohumol (3), and xanthohumol E (4) were isolated from Humulus hipulhts (hop<smiles>COc1cc(O)c(CC=C(C)C)c(O)c1C(=O)/C=C/c1ccc(O)cc1</smiles>
xanthohumol (1) desmethylxanthohuumol (2)<smiles>COc1cc(OC)c(C(=O)/C=C/c2ccc(O)cc2)c(O)c1CC=C(C)C</smiles>

4'-O-methylxanthohumol (3)<smiles>CC(C)=CCc1c(O)c2c(c(C(=O)C=Cc3ccc(O)cc3)c1O)OC(C)(C)C=C2</smiles>

Figure 1. Selected naturally occurring prenylated chalcones plant). which are cultivated in virtually all temperate zones in the world (Figure 1). ${ }^{11}$ This plant is widely used in the brewing industry to add bittemess and aroma to beer. ${ }^{12}$ These compounds have been found to have a variety of interesting biological activities. In particular, they have been shown to be potential anti-proliferative ${ }^{13}$ and cancer chemopreventive agents ${ }^{14}$ with broad-spectrum for the treatment of both breast and prostate cancers. They have been also shown to possess therapeutic utility, including homonal activity for the treatment of osteoporosis ${ }^{15}$ an antioxidant for the treatment of atherosclerosis. ${ }^{16}$ and inhibitory activity against HIV-I. ${ }^{17}$ In addition. these compounds have also significant anti-inflammatory ${ }^{18}$ and antimutagenic activities. ${ }^{1+4}$ Synthetic approaches to xanthohumol (1). ${ }^{19}$ desmethylxanthohumol (2). ${ }^{211}$ and $4^{\prime}-O$-methylxanthohumol $(3)^{21}$ have been already reported by other groups. However. the total synthesis of xanthohumol $E$ (4) has not been reported thus far. Sericone (5), a regioisomer of xanthohumol $E(t)$, was isolated from another plant, Mindulea serice ${ }^{2 \%}$ One synthetic approach to sericone (5) was reported by Diller. but this synthetic method suffers from the disadvantages of having many reaction steps. ${ }^{\text {t2 }}$ This range of important biological properties and activities has stimulated research into the synthesis of naturally occurring $4^{\prime}-0$-methylxantholumol (3), xanthohumol $E(4)$, and sericone (5).

\section{Results and Discussion}

Recently, we developed a new and useful methodology for preparing a variety of benzopyrans using ethylenediamine diacetate-catalyzed reactions of resorcinols or naphthols to $\alpha . \beta$-unsaturated aldelydes.$^{23}$ Using this methodology as a key step. convergent synthetic routes have also been developed to provide biologically interesting natural products with benzopyran moiety ${ }^{24}$ As part an of ongoing study into the synthetic efficacy of these two methodologies, this paper reports a new synthetic route for biologically interesting prenylated chalcone natural products, $4 '-j$-methylxantholumol (3), xantholumol E (4), and sericone (5). 
<smiles>CC(=O)c1c(O)cc(O)cc1O</smiles>

6<smiles>CCOc1ccc(/C=C/C(=O)c2c(OC)cc(OC)c(CC=C(C)C)c2O)cc1</smiles><smiles>COc1ccc(C=O)cc1</smiles>

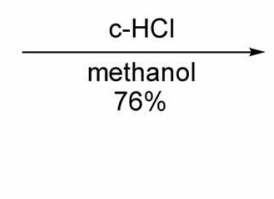<smiles>COc1cc(OC)c(C(=O)/C=C/c2ccc(O)cc2)c(O)c1CC=C(C)C</smiles>

Scheme 1

The synthesis of natural 4'-O-methylxantholuumol (3) was first attempted starting from 2,4.6-trihydroxyacetophenone (6). as shown in Scheme 1. Previous stidies reported that $C$ prenylation can be achieved with prenyl bromide in the presence of either potassium hydroxide ${ }^{2}$ or potassium carbonate. ${ }^{ \pm 5}$ Under these conditions. the desired product 7 was obtained in $30-34 \%$ yield. A better yield was obtained with DBU. A reaction of compound 6 with prenyl bromide in the presence of 1.1 equiv of $\mathrm{DBU}$ in THF at room temperature for $48 \mathrm{~h}$ gave compound 7 in $40 \%$ yield. Treatment of compound 7 with 2 equiv of dimethyl sulfate in the presence of potassium carbonate in acetone at room temperature for $t$ h gave product $\mathbf{8}$ in $65 \%$ yield. An aldol reaction was next attempted to complete the synthesis of natural 4'-O-methylxantholnumol (3). The condensation of conpound 8 with benzaldehyde 9 protected as a SEM ether in an ethanolic $\mathrm{KOH}$ solution at room temperature for $48 \mathrm{~h}$ afforded product $\mathbf{1 0}$ in $75 \%$ yield. Deprotection of compound $\mathbf{1 0}$ through a treatment with $\mathrm{c}-\mathrm{HCl}$ in methanol at room temperature for $\mathrm{I}$ h gave the natural product 3 in $76 \%$ yield. The spectral data of the synthetic material 3 were in agreement with those reported in the literature ${ }^{\geq 6}$

Next. the total synthesis of xanthohumol E (4) and sericone (5) was attempted. The strategy is depicted in Scheme 2. Xanthohumol E (4) and sericone (5) can be prepared from the base-catalyzed aldol reactions of the corresponding compounds 11 and 12 with the benzaldehyde 9 protected as a SEM ether. The crucial intermediates 11 and 12 could be generated from prenylated phloroacetophenone 7 through ethỵlenediamine diacetate-catalyzed benzopyran formation reactions

Schemes 3-5 show the reaction pathway for the synthesis of natural xanthohumol $\mathrm{E}(4)$ and sericone (5). The cycloadditon reaction of prenylated compound 7 described above was first attempted (Scheme 3). A reaction of compound 7 with 3-methyl-2-butenal in the presence of $20 \mathrm{~mol} \%$ of ethylenediamine diacetate (EDDA) in methylene chloride at room temperature for $12 \mathrm{~h}$ provided the products 11 and 12 in 50 and $43 \%$ yield respectively. These two compounds were readily separated by column chromatography and assigned by a comparison with other compounds including<smiles>C=CC(=O)c1c(O)c(CC=C(C)C)c(O)c(C=C)c1Oc1c(O)c2c(c(C(=O)C=C)c1O)OC(C)(C)C=C2</smiles>

Scheme 2<smiles>CC(=O)c1c(O)cc(O)c(CC=C(C)C)c1O</smiles><smiles>CC(=O)c1c(CC=C(C)C)c(O)c(O)c2c1OC(C)(C)C=C2</smiles><smiles>CC(=O)c1c(O)c2c(c(CC=C(C)C)c1O)OC(C)(C)C=C2</smiles>

Scheme 3

these types of benzopyran skeletons as an angular and linear form ${ }^{77}$ The chemical shifts of the two vinylic protons on the py ranyl ring of compound 11 were more upfield than those of compound 12. The ${ }^{1} \mathrm{H}$ NMR spectrum of compound 11 showed two viny lic protons on the pyranyl ring at $\delta 6.52(\mathrm{~J}=$ $9.9 \mathrm{~Hz})$ and $5.4 \mathrm{l}(J=9.9 \mathrm{~Hz})$, whereas compound 12 showed two vinylic protons at $\delta 6.60(J=9.9 \mathrm{~Hz})$ and $5.43(J=$ $9.9 \mathrm{~Hz}$ ).

Next. further reactions shown in Scheme 4 were carried out to complete the synthesis of xanthohumol $\mathrm{E}$ (4). Protection of compound 11 with 2.5 equiv of MOMCl in the presence of disopropylethylamine in methỵlene chloride at room temperature for 12 h provided compound 13 in $90 \%$ yield. Compound 13 was then condensed with the corresponding aldehyde 9 in ethanolic $\mathrm{KOH}$ solution at room temperature for $48 \mathrm{~h}$ to give pyranochalcone 14 in $83 \%$ 


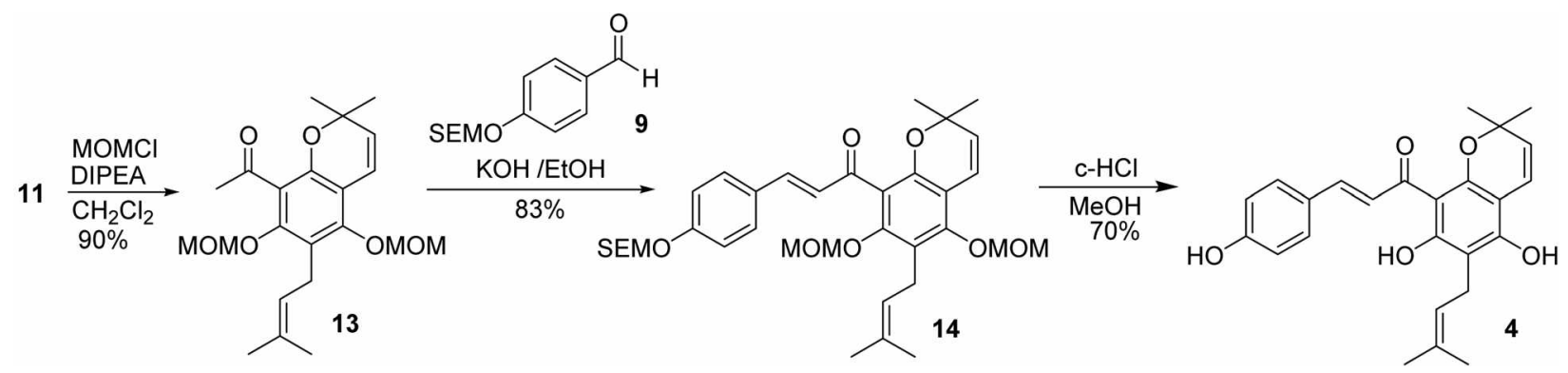

Scheme 4

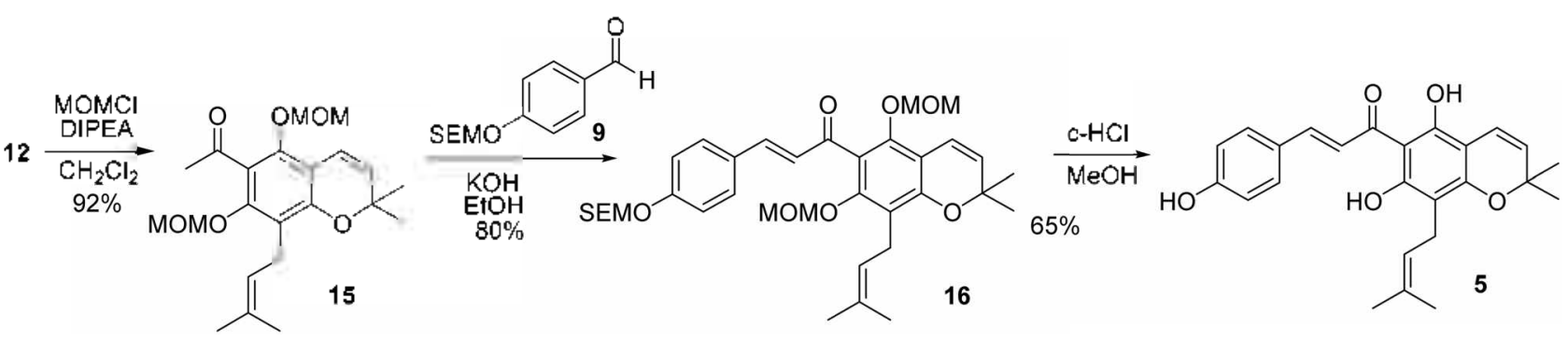

Scheme 5

yield. Attempts to deprotect compound 14 with TBAF or magnesium bromide diethyl etherate ${ }^{3 s}$ did not give the desired natural product 4 . Fortunately, all the protecting groups were easily removed by a treatment with $\mathrm{c}-\mathrm{HCl}$ in methanol at room temperature for $I \mathrm{~h}$ to yield xanthohumol $E$ (4) in $70 \%$ yield. The spectroscopic data of synthetic material 4 were in agreement with those reported in the literature. 39

Additional reactions were carried out to synthesize sericone (5) as shown in Scheme 5. A reaction of compound 12 with 2.5 equiv of $\mathrm{MOMCl}$ gave product $15 \mathrm{in} 92 \%$ yield. Condensation of compound $\mathbf{1 5}$ with benzaldehyde 9 in $\mathrm{KOH}$ at room temperature for $48 \mathrm{~h}$ followed by cleavage of the $\mathrm{MOM}$ and SEM ethers with $\mathrm{c}-\mathrm{HCl}$ in methanol at room temperature for $1 \mathrm{~h}$ afforded sericone (5) in $52 \%$ yield (2steps).

In conclusion. a new and concise synthetic route for biologically interesting prenylated pyranochalcone natural products. 4'-O-methylxanthohumol (3). xanthohumol E (4). and sericone (5) was developed starting from 2,4,6-tribydroxyacetophenone (6). The key strategies for 4'-O-methylxanthohumol (3) involve the insertion of a preyl group onto the aryl ring and base-catalyzed aldol reactions. The key strategies for xantholumol E (4) and sericone (5) involve benzopyran fommation reactions and base-catalyzed aldol reactions.

\section{Experimental}

All the experiments were carried out in a nitrogen atmosphere. Merck precoated silica gel plates (Art. 5554) with a fluorescent indicator were used for analytical TLC. Flash columun chromatography was performed using silica gel 9385 (Merck). The ${ }^{1} \mathrm{H}$ NMR and ${ }^{13} \mathrm{C}$ NMR spectra were recorded on a Bruker Model ARX $(300)$ and $75 \mathrm{MH} \iota$, respec- tively) spectrometer in $\mathrm{CDCl}_{3}$ and benzene $\mathrm{d}_{6}$ as the solvent chemical shift. The IR spectra were recorded on a Jasco FTIR 5300 spectrophotometer. The HRMS and MS spectra were carried out at the Korea Basic Science Institute.

2,4,6-Trihydroxy-3-prenylacetophenone (7). A mixture of phloracetophenone (6) (2.02 g. $12.0 \mathrm{mmol})$. prenyl bromide ( $1.79 \mathrm{~g} .12 .0 \mathrm{mmol}$ ). and DBU (2.01 g. $13.2 \mathrm{mmol})$ in dry THF $(50 \mathrm{~mL})$ was stirred at room temperature for 48 h. Addition of $2 \mathrm{~N} \mathrm{HCl}$ solution $(50 \mathrm{~mL})$, and extraction with ethyl acetate $(3 \times 50 \mathrm{~mL})$, washing with brine $(50 \mathrm{~mL})$, drying over $\mathrm{MgSO}_{4}$ and removal of the solvent followed by flash column chromatography on silica gel using hexane/ ethyl acetate $(5: 1)$ gave $7(1.13 \mathrm{~g} .40 \%)$ as a solid: mp 166$167^{\circ} \mathrm{C}$. ${ }^{1} \mathrm{H}$ NMR $\left(300 \mathrm{MHz} . \mathrm{CDCl}_{3}\right) \delta 5.82(\mathrm{lH}, \mathrm{s}), 5.22$ $(1 \mathrm{H}, \mathrm{t} J=7.0 \mathrm{~Hz}), 3.33(2 \mathrm{H} . \mathrm{d} J=7.0 \mathrm{~Hz}) .2 .64(3 \mathrm{H}, \mathrm{s})$, $1.80(3 \mathrm{H}, \mathrm{s}), 1.75(3 \mathrm{H}, \mathrm{s}):{ }^{13} \mathrm{C} \operatorname{NMR}\left(75 \mathrm{MHz}, \mathrm{CDCl}_{2}\right) \delta$ 203.6, 163.5. 162.1, 160.5. 132.4, 122.9. 106.5, 104.9.94.7. 32.8, 25.9. 21.4, 17.9: IR (KBr) 3418, 1634, 1402, 1370 . 1285. 1235. 1150. 1073.986.818 $\mathrm{cm}^{-1}$, EIMS mz (\%) 236 $\left(\mathrm{M}^{-}, 60\right), 221(29), 193(29) .181(100), 165(40), 153(17)$. 69 (24): HRMS $m z\left(\mathrm{M}^{-}\right)$calcd for $\mathrm{C}_{13} \mathrm{H}_{16} \mathrm{O}_{4}: 236.1049$. Found: 236.1050 .

2-Hydroxy-4,6-dimethoxy-3-prenylacetophenone (8). $\mathrm{K}_{2} \mathrm{CO}_{3}(1.87 \mathrm{~g}, 13.50 \mathrm{mmol})$ was added to a solution of 7 $(0.64 \mathrm{~g} .2 .7 \mathrm{mmol})$ in acetone $(10 \mathrm{~mL})$ at room temperature. Dinethyl sulfate $(0.68 \mathrm{~g} .5 .4 \mathrm{mmol})$ was then added dropwise. and the mixture was stirred at room temperature for $4 \mathrm{~h}$. A saturated $\mathrm{NH}_{4} \mathrm{Cl}$ solution $(50 \mathrm{~mL})$ was added and the mixture was extracted with ethyl acetate $(30 \mathrm{~mL} \times 3)$. The organic layer was dried over $\mathrm{MgSO}_{4}$ and the solvent was removed under reduced pressure to leave an oily residue. The residue was then purified by column cluromatography on silica gel using hexane/ethyl acetate $(15: 1)$ to give the product $8(0.46 \mathrm{~g} .65 \%)$ as a solid: $\mathrm{mp} 107-108{ }^{\circ} \mathrm{C} ;{ }^{1} \mathrm{H}$ NMR $\left(300 \mathrm{MHz}_{2} \mathrm{CDCl}_{3}\right) \delta 13.95(\mathrm{lH}, \mathrm{s}), 5.89(1 \mathrm{H}, \mathrm{s}), 5.15$ (lH. t. 
$J=6.9 \mathrm{~Hz}) .3 .84(6 \mathrm{H} . \mathrm{s}) .3 .22(2 \mathrm{H}, \mathrm{d}, J=6.9 \mathrm{~Hz}) .2 .55(3 \mathrm{H}$. s). $1.74(3 \mathrm{H}, \mathrm{s}), 1.63(3 \mathrm{H} . \mathrm{s}):{ }^{13} \mathrm{C} \mathrm{NMR}\left(75 \mathrm{MHz}, \mathrm{CDCl}_{3}\right) \delta$ 203.2. 163.3, 163.1. 161.6, 131.1. 122.6, 109.4, 105.7. 85.7. $55.3,55.2,33.0 .25 .7,21.1,17.6$; IR (KBr) 3454, 2909. 1624. $1595,1460,1420.1275 .1211 .1123 .893 .791 \mathrm{~cm}^{-1}$. EIMS $m z(\%) 264\left(\mathrm{M}^{-} .90\right), 250(16), 249(100) .221(70)$. 209 (85). 193 (40). 191 (16). 181 (22). 69 (15).

Compound 10. To a solution of $8(0.13 \mathrm{~g}, 0.5 \mathrm{mmol})$ in ethanol $(10 \mathrm{~mL})$ was added potassim hydroxide $(0.14 \mathrm{~g} .2 .5$ mmol $)$ and aldehyde $9(0.15 \mathrm{~g}, 0.6 \mathrm{mmol})$ at room temperature. The reaction mixture was stirred for $48 \mathrm{~h}$ at room temperature. Evaporation of ethanol and extraction with ethyl acetate $(3 \times 50 \mathrm{~mL})$, washing with $2 \mathrm{~N} \mathrm{HCl}$ solution $(30 \mathrm{~mL})$ and brine $(30 \mathrm{~mL})$, drying over $\mathrm{MgSO}_{4}$ and removal of the solvent followed by flash column chromatography on silica gel using hexane/ethyl acetate (20:1) gave $10(0.19 \mathrm{~g}$. $75 \%$ ) as an oil: ${ }^{1} \mathrm{H} \mathrm{NMR}\left(300 \mathrm{MHz} . \mathrm{CDCl}_{3}\right) \delta 14.15(1 \mathrm{H} . \mathrm{s})$. $7.77(\mathrm{lH} . \mathrm{d}, J=15.8 \mathrm{~Hz}), 7.72(1 \mathrm{H} . \mathrm{d}, J=15.8 \mathrm{~Hz}), 7.52$ $(2 \mathrm{H}, \mathrm{d}, J=8.7 \mathrm{~Hz}) .7 .01(2 \mathrm{H}, \mathrm{d}, J=8.7 \mathrm{~Hz}) .5 .93(\mathrm{lH} . \mathrm{s})$. $5.24(2 \mathrm{H} . \mathrm{s}), 5.19(1 \mathrm{H}, \mathrm{t}, J=7.0 \mathrm{~Hz}), 3.9 \mathrm{l}(3 \mathrm{H}, \mathrm{s}) .3 .87(3 \mathrm{H}$. s). $3.78(2 \mathrm{H} . \mathrm{t}, J=8.6 \mathrm{~Hz}) .3 .27(2 \mathrm{H} . \mathrm{d}, J=7.0 \mathrm{~Hz}), 1.76$ $(3 \mathrm{H}, \mathrm{s}) .1 .66(3 \mathrm{H} . \mathrm{s}), 0.94(2 \mathrm{H}, \mathrm{t}, J=8.6 \mathrm{~Hz}),-0.0 \mathrm{l}(9 \mathrm{H} . \mathrm{s})$ : ${ }^{13} \mathrm{C}$ NMR (75 $\left.\mathrm{MHz}, \mathrm{CDCl}_{3}\right) \delta 192.9 .164 .0,163.1 .161 .1$. $159.0 .141 .8,131.3,129.8,129.1,125.8 .122 .7,116.3$. 109.9. 106.3. 92.6. 86.3. 66.4, 55.7, 55.4, 25.8. 21.3. 18.0. $17.7,-1.5:$ IR (neat) $2938,1607,1514.1169,1116 \mathrm{~cm}^{-1}$ : EIMS $m z(\%) 498\left(\mathrm{M}^{-}, 34\right), 400(16), 397(27) .372(50)$. $371(52), 233$ (22), 207924$), 193(47), 192$ (28). 181 (36). $180(15) .179(53) .73(100)$

4'-O-Methylxanthohumol (3). To a solution of $\mathbf{1 0}(0.16$ g. $0.31 \mathrm{mmol})$ in methanol $(10 \mathrm{~mL})$ was added $\mathrm{c}-\mathrm{HCl}(5$ drops) and the reaction mixture was stirred at room temperature for $\mathrm{l} \mathrm{h}$. The reaction mixture was diluted with water $(20 \mathrm{~mL})$. and extracted with EtOAc $(3 \times 30 \mathrm{~mL})$. The combined organic phases were washed with saturated $\mathrm{NaHCO}_{3}$ solution $(30 \mathrm{~mL})$. water $(30 \mathrm{~mL})$. and dried over $\mathrm{MgSO}_{4}$. Removal of solvent at reduced pressure left an oily residue. which was then purified by column chromatography on silica gel using hexane/ethyl acetate (2:1) to give $3(0.08 \mathrm{~g}$. $76 \%$ ) as a solid: $\mathrm{mp} 152-153{ }^{\circ} \mathrm{C} ;{ }^{1} \mathrm{H}$ NMR $(300 \mathrm{MHz}$. $\left.\mathrm{CDCl}_{\mathrm{s}}\right) \delta 7.75(\mathrm{lH} . \mathrm{d}, J=15.8 \mathrm{~Hz}), 7.70(1 \mathrm{H}, \mathrm{d}, J=15.8$ $\mathrm{Hz}) .7 .45(2 \mathrm{H}, \mathrm{d} J=8.7 \mathrm{~Hz}) .6 .84(2 \mathrm{H}, \mathrm{d} J=8.7 \mathrm{~Hz}) .5 .97$ $(1 \mathrm{H} . \mathrm{s}) .5 .18(1 \mathrm{H}, \mathrm{t} J=6.9 \mathrm{~Hz}) .3 .91(3 \mathrm{H} . \mathrm{s}) .3 .87(3 \mathrm{H} . \mathrm{s})$. $3.27(2 \mathrm{H}$. d. $J=6.9 \mathrm{~Hz}) .1 .76(3 \mathrm{H}, \mathrm{s}) .1 .65(3 \mathrm{H} . \mathrm{s}):{ }^{13} \mathrm{C} \mathrm{NMR}$ $\left(75 \mathrm{MHz}, \mathrm{CDCl}_{3}\right) \delta 193.1,163.9,163.3,162.3 .161 .3,157.7$. 131.5. 130.3, 128.1. 125.2. 122.6, 115.9, 109.9. 106.4, 86.4. 55.8. 55.5. 25.8. 21.4 14.1: IR (KBr) 3368. 1607. 1512. 1416. 1329. 1227. 1169.1138. 1117. 978. $831.733 \mathrm{~cm}^{-1}$ : EIMS $m z(\%) 368\left(\mathrm{M}^{-}, 80\right), 358(25), 325(73), 313(27)$. 261 (16). $248(15) .233$ (77), 219 (17), 205 (45), 193 (100). $181915) .147(22) .119(24) .107(24) .91(44) .77$ (21). 65 (26): HRMS $m z\left(\mathrm{M}^{-}\right)$calcd for $\mathrm{C}_{2\lrcorner} \mathrm{H}_{\unlhd} \mathrm{O}_{\Sigma}: 368.4230$. Found: 368.4232 .

1-(5,7-Dihydroxy-2,2-dimethyl-6-prenyl-2 $H$-chromen8-yl)ethanone (11) and 1-(5,7-dihydroxy-2,2-dimethyl-8prenyl-2H-chromen-6-yl)ethanone (12). To a solution of 7 $(0.66$ g. $2.8 \mathrm{mmol})$ and 3-methyl-2-butenal $(0.47$ g. 5.6 mumol) in methylene chloride $(20 \mathrm{~mL})$ was added etlylenediamine diacetate $(0.10 \mathrm{~g} .0 .6 \mathrm{mmol})$ at room temperature. The reaction mixture was stirred at room temperature for 12 h. Water $(50 \mathrm{~mL})$ was added and the solution was extracted with metlyylene chloride $(3 \times 50 \mathrm{~mL})$. Evaporation of solvent and purification by colunn chromatography on silica gel using hexane/ethyl acetate $(10: 1)$ gave $11(0.42 \mathrm{~g} .50 \%)$ and 12 (0.36 g. 43\%). Compound 11: $\mathrm{mp} 80-81{ }^{\circ} \mathrm{C}$ : ${ }^{1} \mathrm{H}$ NMR $\left(300 \mathrm{MHz}, \mathrm{CDCl}_{3}\right) \delta 14.10(\mathrm{lH} . \mathrm{s}) .6 .52(\mathrm{lH} . \mathrm{d} . J=9.9 \mathrm{~Hz})$, $6.36(\mathrm{lH}$, br s). $5.4 \mathrm{l}(\mathrm{lH}, \mathrm{d}, J=9.9 \mathrm{~Hz}), 5.23$ (lH. t. $J=7.0$ Hz). $3.35(2 \mathrm{H}, \mathrm{d} . J=7.0 \mathrm{~Hz}) .2 .65(3 \mathrm{H}, \mathrm{s}), 1.8 \mathrm{l}(3 \mathrm{H} . \mathrm{s}) .1 .75$ (3H. s), $1.46(6 \mathrm{H} . \mathrm{s}) ;{ }^{13} \mathrm{C}$ NMR $\left(75 \mathrm{MHz}, \mathrm{CDCl}_{3}\right) \delta 203.3$, $160.5,156.7,154.9 .125 .4,124.7$. 116.4. 116.1, 105.5. 102.3. 102.1, 78.2. 33.2, 28.3. 28.0: IR (KBr) 2976, 1601, 1464, 1364. 1283. 1196, 1140. 1003. $883,729 \mathrm{~cm}^{-1}$. EIMS m/z (\%) $302\left(\mathrm{M}^{-}, 42\right) .288(9) .287(48), 247(10) .232(15) .231$ (100), 213 (17). Compound 12: ${ }^{1} \mathrm{H}$ NMR ( $\left(300 \mathrm{MHz} . \mathrm{CDCl}_{3}\right)$ $\delta 12.69$ (lH. s). 7.79 (lH. br s). $6.60(1 \mathrm{H}, \mathrm{d}, J=9.9 \mathrm{~Hz}$ ), $5.43(\mathrm{lH} . \mathrm{d} . J=9.9 \mathrm{~Hz}) .5 .15(\mathrm{lH}, \mathrm{t} . J=7.0 \mathrm{~Hz}) .3 .29(2 \mathrm{H}$. d. $J=7.0 \mathrm{~Hz}), 2.61(3 \mathrm{H} . \mathrm{s}), 1.80(3 \mathrm{H}, \mathrm{s}), 1.73(3 \mathrm{H}, \mathrm{s}), 1.38$ (6H. s). ${ }^{13} \mathrm{C}$ NMR (75 MHz, $\left.\mathrm{CDCl}_{3}\right) \delta 203.3,159.1$. 157.6. 156.8, 134.9. 125.0, 121.1. 115.6. 105.2, 104.6, 101.8. 77.2. 32.4. 27.6, 25.3, 20.8, 17.4; IR (neat) 2921. 1613. 1453. $1126,802 \mathrm{~cm}^{-1}$; EIMS $m z(\%) 302\left(\mathrm{M}^{-} .29\right), 288(10), 287$ (52). 247 (15). 232 (14). 231 (100). 213 (13).

1-(5,7-Bismethoxymethoxy-2,2-dimethyl-6-prenyl-2Hchromen-8-yl)ethanone (13). Methoxymethyl chloride $(0.20 \mathrm{~g} .2 .5 \mathrm{mmmol})$ was added to a solution of $11(0.30 \mathrm{~g}, 1.0$ munol) and $N, N$-diisopropy lethylamine $(0.65 \mathrm{~g} .5 .0 \mathrm{mmol})$ in dry methylene chloride $(20 \mathrm{~mL})$ at room temperature. The reaction mixture was stirred at room temperature for $12 \mathrm{~h}$, and then water $(40 \mathrm{~mL})$ was added. The reaction mixture was extracted with methylene chloride $(3 \times 30 \mathrm{~mL})$ and the combined organic extracts were washed with saturated $\mathrm{NH}_{4} \mathrm{Cl}$ solution $(30 \mathrm{~mL})$. water $(30 \mathrm{~mL})$, dried $\left(\mathrm{MgSO}_{4}\right)$, and evaporated in vacuo. Flash clromatograply on silica gel using hexane/ethyl acetate (7:1) afforded $13(0.35 \mathrm{~g} .90 \%)$ as an oil: ${ }^{1} \mathrm{H}$ NMR $\left(300 \mathrm{MHz}, \mathrm{CDCl}_{3}\right) \delta 6.48(1 \mathrm{H}, \mathrm{d} . J=9.9$ Hz). $5.57(1 \mathrm{H}$. d. $J=9.9 \mathrm{~Hz}) .5 .11$ (1H. t. $J=7.0 \mathrm{~Hz}) .4 .87$ $(2 \mathrm{H} . \mathrm{s}) .486(2 \mathrm{H} . \mathrm{s}) .3 .44(6 \mathrm{H} . \mathrm{s}) .3 .24(2 \mathrm{H} . \mathrm{d} . J=7.0 \mathrm{~Hz})$. $2.50(3 \mathrm{H}, \mathrm{s}), 1.72(3 \mathrm{H}, \mathrm{s}) .1 .63(3 \mathrm{H}, \mathrm{s}): 1.37(6 \mathrm{H}, \mathrm{s}):{ }^{12} \mathrm{C}$ $\operatorname{NMR}\left(75 \mathrm{MHz}, \mathrm{CDCl}_{3}\right) \delta 201.8,153.3,152.7,148.5,131.2$, $129.6,122.5,120.0,116.8$. 111.9, 101.2. 100.0. 76.4, 57.6, 32.7. 27.7, 25.7, 22.8, 17.9: IR (neat) 2975. 2928. 1697. 1642. 1588, 1452, 1373, 1238, 1213, 1124, 1059. 1023. 991. 932. 894, 800, $753 \mathrm{~cm}^{-1}$; EIMS $m z(\%) 390\left(\mathrm{M}^{+} .22\right), 346$ (34). 331 (18). 302 (11). 301 (52). 299 (18). 247 (15). 246 (16). 245 (12). 232 (14). 231 (100). 213 (11).

Compound 14. To a solution of $13(0.26 \mathrm{~g} .0 .7 \mathrm{mmol})$ in ethanol $(10 \mathrm{~mL})$ was added potassium hydroxide $(0.19 \mathrm{~g} .3 .3$ mumol) and aldehy'de $9(0.25 \mathrm{~g} .1 .0 \mathrm{mmol})$ at room temperature. The reaction mixture was stirred for $48 \mathrm{~h}$ at room temperature. Evaporation of ethanol and extraction with ethyl acetate $(3 \times 50 \mathrm{~mL})$. washing with $2 \mathrm{~N} \mathrm{HCl}$ solution $(30 \mathrm{~mL})$, water $(30 \mathrm{~mL})$, and brine $(30 \mathrm{~mL})$. drying over $\mathrm{MgSO}_{4}$ and removal of the solvent followed by flash column chromatography on silica gel using hexane/ethyl acetate 
(10:1) gave $1+(0.3+\mathrm{g} .83 \%)$ as an oil: ${ }^{1} \mathrm{H}$ NMR $(300 \mathrm{MHz}$. $\left.\mathrm{CDCl}_{3}\right) \delta 7.47(2 \mathrm{H}, \mathrm{d}, J=8.8 \mathrm{~Hz}), 7.39(\mathrm{lH} . \mathrm{d}, J=16.0 \mathrm{~Hz})$. $7.00(2 \mathrm{H} . \mathrm{d} . J=8.8 \mathrm{~Hz}), 6.92(\mathrm{lH} . \mathrm{d} . J=16.0 \mathrm{~Hz}), 6.54(\mathrm{lH}$. d. $J=9.9 \mathrm{~Hz}) .5 .59(\mathrm{lH} . \mathrm{d} . J=9.9 \mathrm{~Hz}) .5 .21(2 \mathrm{H}, \mathrm{s}) .5 .15$ $(1 \mathrm{H}, \mathrm{t} . J=7.0 \mathrm{~Hz}), 4.88(2 \mathrm{H} . \mathrm{s}) .4 .87(2 \mathrm{H}, \mathrm{s}) .3 .81(2 \mathrm{H}, \mathrm{t} . J=$ $8.4 \mathrm{~Hz}$ ). $3.4 \mathrm{l}$ (3H. s). 3.40 (3H. s). 3.30 ( $2 \mathrm{H.d} . J=7.0 \mathrm{~Hz}$ ). $1.75(3 \mathrm{H}, \mathrm{s}), 1.66(3 \mathrm{H} . \mathrm{s}), 1.40(6 \mathrm{H} . \mathrm{s}), 0.92(2 \mathrm{H} . \mathrm{t} . J=8.4$ $\mathrm{Hz}),-0.03(9 \mathrm{H}, \mathrm{s}) ;{ }^{13} \mathrm{C}$ NMR $\left(75 \mathrm{MHz} . \mathrm{CDCl}_{3}\right) \delta 194.0$. 159.8. 153.9. 149.8, 144.6, 131.6. 130.5, 129.9, 128.7. 127.2. 123.2, 122.9. 120.5, 117.5, 116.8. 112.5. 101.4, 101.2. $93.0,76.9 .66 .9 .58 .1,58.0 .28 .3,26.2 .23 .4,18.4 .-1.0$. IR (neat) $2955,1641.1595,1510.1236 .1161,1092.993 .837$ $\mathrm{cm}^{-1}$, EIMS mz (\%) $624\left(\mathrm{M}^{+}, 38\right), 609(2 \mathrm{l}), 580(18), 579$ (15), $577(17), 519(10) .419$ (8), 330 (29). 315 (25). 301 (22), 286 (19), 285 (100). 271 (60). 269 (27). 243 (16), 231 (15). $219(54) .215$ (33). 201 (15), 73 (74).

Xanthohumol E (4). To a solution of $1+(0.21$ g. 0.3 mmol) in methanol $(10 \mathrm{~mL})$ was added $\mathrm{c}-\mathrm{HCl}$ (5 drops) and the reaction mixture was stirred at room temperature for $1 \mathrm{~h}$. The reaction mixture was diluted with water $(20 \mathrm{~mL})$, and extracted with EtOAc $(3 \times 30 \mathrm{~mL})$. The conbined organic phases were washed with saturated $\mathrm{NaHCO}_{3}$ solution, water $\left(30 \mathrm{~mL}\right.$ ), and dried over $\mathrm{MgSO}_{4}$. Removal of solvent at reduced pressure left an oily residue, which was then purified by colunn chromatography on silica gel using hexane/ ethyl acetate (2:1) to give $+(0.09 \mathrm{~g} .70 \%)$ as an oil; ${ }^{3} \mathrm{H}$ NMR $\left(300 \mathrm{MHz}\right.$, benzene $\left.-d_{6}\right) \delta \mathrm{l} 4.56(\mathrm{lH}$. br s), 7.98 (lH. d. $J=$ $15.6 \mathrm{~Hz}), 7.7 \mathrm{l}(\mathrm{lH} . \mathrm{d}, J=15.6 \mathrm{~Hz}) .7 .46(2 \mathrm{H}, \mathrm{d} . J=8.6 \mathrm{~Hz})$. $6.86(2 \mathrm{H}, \mathrm{d}, J=8.6 \mathrm{~Hz}) .6 .55(1 \mathrm{H} . \mathrm{d} . J=9.9 \mathrm{~Hz}) .6 .42(\mathrm{IH}$. br s). $5.45(1 \mathrm{H} . \mathrm{d} . J=9.9 \mathrm{~Hz}) .5 .27(1 \mathrm{H} . \mathrm{t} . J=7.0 \mathrm{~Hz}) .3 .38$ $(2 \mathrm{H}, \mathrm{d} . J=7.0 \mathrm{~Hz}) .1 .81(3 \mathrm{H} . \mathrm{s}) .1 .76(3 \mathrm{H}, \mathrm{s}), 1.51(6 \mathrm{H}, \mathrm{s})$ : ${ }^{13} \mathrm{C}$ NMR (75 MHz. DMSO- $\left.d_{6}\right) \delta 191.8,163.3 .160 .1,157.2$. $153.6 .142 .5,130.3 .130 .2,125.9 .125 .0,123.5 .122 .8,117.1$, 116.0. 108.3, 105.0, 102.5. 77.1, 27.2, 25.4. 21.1. 17.7; IR (neat) $3395,1604,1512,1443,1346,1167,831 \mathrm{~cm}^{-1}$; EIMS $m z(\%) 406\left(\mathrm{M}^{-}, 81\right) .405(19), 392(24), 391(93) .368(24)$. 351 (16). $286(15), 285(36), 271$ (46), $243(25), 231$ (28). $230(15) .229$ (16). 215 (100). 147 (17). 97 (15). 91 (17). 83 (17). 81 (15). 71 (16). 69 (19): HRMS $m z\left(\mathrm{M}^{+}\right)$calcd for $\mathrm{C}_{\unlhd 5} \mathrm{H}_{\triangle 6} \mathrm{O}_{s}: 406.4709$. Found: 406.4707 .

1-(5,7-Bismethoxymethoxy-2,2-dimethyl-8-prenyl- $2 \mathrm{H}$ chromen-6-yl)ethanone (15). Methosymethyl chloride $(0.17 \mathrm{~g} .2 .1 \mathrm{mmol})$ was added to a solution of $12(0.26 \mathrm{~g} .0 .8$ mmol) and $N . N$-diisopropylethy lamine $(0.54 \mathrm{~g} .4 .2 \mathrm{mmol})$ in dry methylene chloride $(20 \mathrm{~mL})$ at room temperature. The reaction mixture was stirred at room temperature for $12 \mathrm{~h}$. and then water $(40 \mathrm{~mL})$ was added. The reaction mixture was extracted with methylene chloride $(3 \times 30 \mathrm{~mL})$ and the combined organic extracts were washed with saturated $\mathrm{NH}_{4} \mathrm{Cl}$ solution $(30 \mathrm{~mL})$, water $(30 \mathrm{~mL})$. dried $\left(\mathrm{MgSO}_{4}\right)$. and evaporated in vacuo. Flash chromatography on silica gel using hexane/ethyl acetate (7:1) afforded $15(0.30 \mathrm{~g} .92 \%)$ as an oil: ${ }^{1} \mathrm{H}$ NMR $\left(300 \mathrm{MHz}, \mathrm{CDCl}_{3}\right) \delta 6.46(\mathrm{lH}$. d. $J=9.9$ $\mathrm{Hz}), 5.52(\mathrm{lH} . \mathrm{d}, J=9.9 \mathrm{~Hz}), 5.11(1 \mathrm{H}, \mathrm{t}, J=7.0 \mathrm{~Hz}), 4.87$ $(2 \mathrm{H} . \mathrm{s}) .4 .82(2 \mathrm{H} . \mathrm{s}) .3 .49(3 \mathrm{H} . \mathrm{s}) .3 .39(3 \mathrm{H} . \mathrm{s}) .3 .24$ ( $2 \mathrm{H} . \mathrm{d} . J$ $=7.0 \mathrm{~Hz}) .2 .44(3 \mathrm{H} . \mathrm{s}) .1 .66(3 \mathrm{H} . \mathrm{s}) .1 .56(3 \mathrm{H} . \mathrm{s}): 1.35(6 \mathrm{H}$. s): ${ }^{13} \mathrm{C}$ NMR $\left(75 \mathrm{MHz} . \mathrm{CDCl}_{3}\right) \delta 201.7,154.1,153.5 .149 .6$.
$131.6,129.8,123.8 .123 .0,122.1 .117 .7 .112 .4,101.3 .100 .8$ $76.9,58.0,57.6,35.0 .28 .0 .25 .6 .23 .9 .18 .3$. IR (neat) 2973 . 2928. 1698. 1638. 1586. 1428. 1351. 1281. 1214. 1161, 1072. 1039. 962. $801.753 \mathrm{~cm}^{-1}$ : EIMS $m z(\%) 390\left(\mathrm{M}^{-}\right.$. 28). $346(57) .331(27), 302(14), 301(67), 299$ (23), 285 (13). 247 (15). 246 (16). 245 (12). 232 (14). 231 (100). 213 (II).

Compound 16. To a solution of $15(0.22 \mathrm{~g} .0 .6 \mathrm{mmol})$ in ethanol $(10 \mathrm{~mL})$ was added potassium hydroxide $(0.16 \mathrm{~g}$. $2.9 \mathrm{mmol})$ and aldelyde $9(0.22 \mathrm{~g} .0 .9 \mathrm{mmol})$ at room temperature. The reaction mixture was stirred for $48 \mathrm{~h}$ at room temperature. Evaporation of ethanol and extraction with ethyl acetate $(3 \times 50 \mathrm{~mL})$, washing with $2 \mathrm{~N} \mathrm{HCl}$ solution $(30 \mathrm{~mL})$. water $(30 \mathrm{~mL})$. and brine $(30 \mathrm{~mL})$, drying over $\mathrm{MgSO}_{4}$ and removal of the solvent followed by flash column chromatography on silica gel using hexane/ethyl acetate $(10: 1)$ gave $16(0.29 \mathrm{~g}, 80 \%)$ as an oil: ${ }^{1} \mathrm{H}$ NMR $(300$ $\left.\mathrm{MHz}, \mathrm{CDCl}_{3}\right) \delta 7.43$ (2H. d, $J=8.7 \mathrm{~Hz}$ ). 7.32 (lH. d, $J=$ $16.0 \mathrm{~Hz}) .6 .97(2 \mathrm{H}, \mathrm{d} . J=8.7 \mathrm{~Hz}) .6 .86(1 \mathrm{H}, \mathrm{d} . J=16.0 \mathrm{~Hz})$, 6.52 (lH. d. $J=9.9 \mathrm{~Hz}) .5 .52(\mathrm{lH}$. d. $J=9.9 \mathrm{~Hz}) .5 .18(2 \mathrm{H}$, s) 5.18 (lH. t. $J=7.0 \mathrm{~Hz}$ ), 4.94 (2H. s), $4.87(2 \mathrm{H} . \mathrm{s}), 3.69$ $(2 \mathrm{H} . \mathrm{t}, J=8.4 \mathrm{~Hz}) .3 .54(3 \mathrm{H} . \mathrm{s}), 3.33(3 \mathrm{H}, \mathrm{s}), 3.32(2 \mathrm{H}, \mathrm{d}, J$ $=7.0 \mathrm{~Hz}) .1 .69(3 \mathrm{H}, \mathrm{s}) .1 .64(3 \mathrm{H} . \mathrm{s}) .1 .29(6 \mathrm{H} . \mathrm{s}), 0.90(2 \mathrm{H}$. t. $J=8.4 \mathrm{~Hz}),-0.06(9 \mathrm{H} . \mathrm{s}) ;{ }^{13} \mathrm{C} \mathrm{NMR}\left(75 \mathrm{MHz}, \mathrm{CDCl}_{3}\right) \delta$ $193.5,159.1,153.5 .149 .5 .144 .1,131.0,129.7 .129 .3$. $128.1,126.7 .123 .3,121.5 .120 .4,117.2 .116 .2,111.9,100.5$, $100.2,92.4,76.1,66.2,57.4 .57 .1 .27 .5,25.5,23.4,17.8$. -1.6 : IR (neat) 2955. 1645. 1599. 1510, 1314, 1236. 1159. 1130, 1092, 1033, 982.860, $835 \mathrm{~cm}^{-1}$. EIMS $m z(\%) 624$ $\left(\mathrm{M}^{-}, 54\right), 609$ (15). 579 (14). 551 (15). 548 (15). 533 (15), 521 (33). 475 (19). 330 (14). 285 (29). 283 (22). 271 (16). 269 (19). 223 (19). 220 (14). 219 (77). 215 (32), 179 (15). 73 $(100)$.

Sericone (5). To a solution of $16(0.21 \mathrm{~g} .0 .3 \mathrm{mmol})$ in methanol $(10 \mathrm{~mL})$ was added $\mathrm{c}-\mathrm{HCl}(5$ drops $)$ and the reaction mixture was stirred at room temperature for $1 \mathrm{~h}$. The reaction mixture was diluted with water $(20 \mathrm{~mL})$. and extracted with EtOAc $(3 \times 30 \mathrm{~mL})$. The combined organic phases were washed with saturated $\mathrm{NaHCO}_{3}$ solution (30 $\mathrm{mL}$ ), water $(30 \mathrm{~mL})$, and dried over $\mathrm{MgSO}_{4}$. Removal of solvent at reduced pressure left an oily residue. which was then purified by column chromatography on silica gel using hexane/ethyl acetate $(2: 1)$ to give $5(0.09 \mathrm{~g} .65 \%)$ as an oil: ${ }^{1} \mathrm{H}$ NMR (300 MHz. benzene- $\left.d_{b}\right) \delta 13.17$ (1H. br s). 7.82 $(\mathrm{lH} . \mathrm{d} . J=15.5 \mathrm{~Hz}), 7.73(\mathrm{lH}, \mathrm{d}, J=15.5 \mathrm{~Hz}) .7 .50(2 \mathrm{H}$. d. $J=8.6 \mathrm{~Hz}) .7 .28(1 \mathrm{H}$. br $\$$ ). $6.83(2 \mathrm{H} . \mathrm{d} . J=8.6 \mathrm{~Hz}) .6 .66$ $(1 \mathrm{H}$. d. $J=9.9 \mathrm{~Hz}), 5.46(1 \mathrm{H} . \mathrm{d} . J=9.9 \mathrm{~Hz}) .5 .19$ (1H. t. $J=$ $7.0 \mathrm{~Hz}) .3 .34(2 \mathrm{H} . \mathrm{d} . J=7.0 \mathrm{~Hz}) .1 .83(3 \mathrm{H} . \mathrm{s}) .1 .77(3 \mathrm{H} . \mathrm{s})$. 1.42 (6H. s): IR (neat) $3387,1605,1514,1443,1169,833$ $\mathrm{cm}^{-1}$; EIMS $m z(\%) 406\left(\mathrm{M}^{-}, 67\right) .404$ (14). 403 (15), 392 (27). 391 (100), 369 (18). $368(36), 351$ (16). 285 (26). 271 (38). 269 (22). 243 (24). 236 (16). 231 (26). 230 (15). 229 (20). $215(85) .151$ (29). 149 (51). 147 (33). 129 (17). 122 (15). 121 (27). $120(20) .119$ (20). 111 (23). 109 (23). 107 (21). 98 (22), 97 (38). 91 (29). 83 (42), 81 (35). 71 (43). 69 (52): HRMS $m z\left(\mathrm{M}^{+}\right)$calcd for $\mathrm{C}_{25} \mathrm{H}_{26} \mathrm{O}_{5}: 406.4709$. Found: 406.4710 . 
Acknowledgments. This research was supported by the Yeungnam University research grants in 208-A-235-021.

\section{References}

1. (a) Shimizu. K.: Kondo, R:: Sakai. K.: Buabarn. S.: Dilokkunanant. U. Phytochentisty 2000. 54. 737. (b) Jayasinghe. L: Rupasinghe. G. K.: Hara. N.: Fujimoto. Y. Phwtochenistry 2006. 67, 1353. (c) Nishimura. R.: Tabata, K: Motoki. A.: Ito, Y: Kimura, Y: Akilisa, T.: Nagai. H: Sakuma, A.: Kohno. H.: Suzuki, T. Biol. Pham. Buhl. 2007. 30, 1878. (d) Rodriguez, R. J: Miranda. C. L.: Stevens. J. F.: Deinzer. M. L.: Buhler. D. R. Food Chemical. Toxicol. 2001.39.437.

2. Narender. T.: Shweta: Tanvir. K.: Rao. M. S.: Srivastava. K.: Puri. S. K. Bioorg. Med. Chem. Lett. 2005. 15. 2453.

3. Enoki, T.: Olnnogi, H.: Naganine. K: Kudo, Y: Sugivama, K.: Tanabe. M.: Kobayashi. E.: Sagawa. H.: Kato, I. J. Agric. Food Chem. 2007. 55.6013.

4. Jayasinghe. L.: Balasooriya. B. A. I. S.: Padmini. W. C.: Hara. N.: Fujimoto. Y. Phwochenistry 2004. 65. 1287.

5. Yin, S: Fan C.-Q: Wang. Y.: Dong L: Yue J.-M. Bioorg Med. Chem. Lett. 2004. 12, 4387.

6. (a) Akihisa. T: Tokluda. H.: Hasegawa, D.: Ukiya M.: Kimura, Y.: Enjo. F.: Suzuki. T.: Nishino. H. J. Nat. Prod. 2006. 69. 38. (b) Akihisa. T.: Tokuda. H.: Ukiya. M.: Iizuka. M.: Schneider. S.: Ogasawara. K.: Mukainaka. T.: Iwatsuki. K.: Suzuki. T.: Nishino. H. Cancer Lett. 2003, 201. 133. (c) Tabata, K.: Motani. K.: Takayanagi. N.: Nishimura. R.: Asami. S.: Kimura. Y.: Ukiya. M.: Hasegawa, D.: Akilisa. T: Suzuki. T. Biol. Pham Bull 2005, 28. 1404

7. Kimura. Y: Baba. K. Int. J. Cancer 2003. 106. 429.

8. Haraguchi. H.: Ishikawa. H.: Mizutani. K.: Tamura. Y.: Kinoshita. T. Bioorg. Ned them. 1998, 6, 339 .

9. Hsieh, H.-K.: Lee. T. H.: Wang, I.-P.: Wang. I.-J: Lin, C.-N. Pham Res. 1998. 15. 39.

10. Sug̣il. M.: Ohkita. M.: Taniguchi. M.: Baba. K.: Kawai. Y.: Tahara. C.: Takaoka. M.: Matsumura. Y. Bio. Pharm. Bull. 2005. 28. 607.

11. Stevens. J. F.: Ivancic. M.: Hsu. V.: Deinzer. M. L. Phwochemistry 1997, 4,1575

12. Stevens, J. F.: Page I. E. Phytochemistry 2004. 65. 1317.

13. Miranda. C. L. Stevens, J. F.: Helmrich. A.: Henderson. M. C.: Rodriguez. R. J.: Yang. Y.-H.: Deinzer. M. L.: Barnes. D. W: Buhler. D. R. Food Chemical. Toxicol. 1999. 37.271

14. (a) Miranda. C. L.: Aponso. G. L. M.: Stevens. J. F.: Deinzer. M. L.: Buller. D. R. Gaffer Left 2000, 149. 21. (b) Miranda. C. L.: Yang. Y.-H.: Henderson. M. C.: Stevens. J. F.: Santana-Rios, G.:
Deitzer. M. L: Buhler. D. R. Dnyg Hetab. Dispos. 2000. 28. 1297. (c) Henderson. M. C.: Miranda. C. L.: Stevens. I. F.: Deitzer. M. L.: Buhler. D. R. Yenobiotica 2000. 30.235.

15. (a) Overk, C. R; Yao. P; Chadwick. L. R.; Nikolic. D.; Sun. Y; Cuendet. M. A.: Deng. Y.: Hedayat. A. S.: Pauli. G. F.: Farnsworth. N. R.; van Breemen, R. B.: Bolton, J. L. J. Agric. Food Chem. 2005. 53. 6246. (b) Miranda. C. L.: Stevens. J. F.: Ivano. V. McCall. M.: Frei. B.: Deinzer. M. L.: Buhler. D. R. J. Agric. Food Chent 2000. 48.3876.

16. Stevens, J. F.; Miranda, C. L.; Frei. B.: Buhler. D. R. Chem. Res. Toxicol. $2003,16,1277$

17. Wang, Q: Ding. Z.-H.: Liu. J.-K; Zheng. Y.-T. Antiviral Res 2004. 6t. 189

18. Gerhäuser. C.: Alt. A.: Heiss. E.: Gamal-Eldeen. A.: Klimo. K.: Knlauft. I.: Neumant1. I.: Scherf. H.-R.: Frank. N.: Bartsch. H.: Becker. H. Hol. Cancer Ther 2002. 1.959

19. Khupse, R. S.; Erhardt, P. W. J. Kat. Prod. 2007. 70, 1507.

20. Diller. R. A.; Riepl. H. M.; Rose. O.: Frias. C.: Henze. G.: Prokop. A. Chent Biodiners. 2005. 2. 1331.

21. Jain. A. C.: Sinha. S. P. Indiam J. Chent Sec. B 1994. 33.317.

22. Zyl. J. J. V.: Rall. G. T. H.: Roux. D. G. J. Chem. Research (S) 1979.97.

23. a) Lee, Y. R.: Choi, J. H.: Yoon. S. H Tetahedron Lett 2005. 46. 7539. (b) Wang. X.: Lee, Y. R. Tetrahedron Lett. 2007. 48,6275 (c) Lee. Y. R.: Kiml. Y. M. Heh: Chim Acta 2007. 90. 2401. (d) Lee. Y. R.: Kim. J. H. Sintett 2007. 2232.

24. (a) Wang. X.: Lee. Y. R. Symthesis 2007. 3044 . (a) Lee. Y. R.: Xia. L. Symthesis $\mathbf{2 0 0 7}, 3240$ (c) Lee, Y. R: Lee. W. K; Noh, S. K. Lyoo. W. S. Synthesis 2006. 853. (d) Lee. Y. R.; Kim. D. H. Synthesis 2006, 603. (e) Lee. Y. R.; Xia, L. Bull Korean Chem. Soc. 2007. 28. 1579. (f) Lee. Y. R.: Wang. X.: Kim. Y. M.: Shim. J. J.: Kiml. B. N.: Hant. D. H. Bull. Konew Chent Soc. 2007. 28. 1735. (g) Lee. Y. R.: Li. X. Bull. Korean Chent. Soc. 2007. 28. 1739. (h) Lee. Y. R.: Wang. X. Bull Korean Chem. Soc. 2007,28 2061. (i) Lee, Y. R.; Xia, L. Tetahedron Lett. 2008. 49, 3283. (j) Lee. Y. R.: Li. X; Kim. J. H. J. Org. Chem in press. (k) Lee, Y. R.: Xia. L. Sinlett. in press.

25. Yan1. Y.G.: Zhan1. Y.: Cao. X.P. Huarue Huebao 2005. 63.1901 26. Sun1. S.: Watanabe. S.: Saito. T. Phwochentistry 1989. 28. 1776.

27. (a) Daikonya A.; Katsuki, S.: Kitanaka, S. Chem. Phom. Bull. 2004. 52, 1326. (b) Daikonya, A.; Katsuli. S.; Wu. J.-B.; Kitanaka. S. Chem. Pham Bull 2002, 50, 1566. (c) Su. C.-R : Kuo. P.C Wang. M.-L.: Liou. M.-T.: Damu. A. G.: Wu. T.-S. J. Nat. Prod. 2003. 66.990

28. Vakalopoulos. A.: Hoffimann. H. M. R. Org. Lett. 2001. 3. 2185.

29. Stevens, J. F.: Taylor, A. W.: Nickerson, G. B.: Ivancic. M.: Henning, J: Haunold, A.; Deinzer. M. L. Plytochenistry 2000. 53.759 . 